\title{
Jointly Optimal Chunk and Power Allocation in Uplink SC-FDMA
}

\author{
Teoman Mert \\ Department of ECE, \\ Istanbul Technical University, \\ Maslak, Istanbul, Turkey \\ tmert@itu.edu.tr
}

\author{
Onur Kaya \\ Department of EEE, \\ Iş1k University, \\ Şile, Istanbul, Turkey \\ onurkaya@isikun.edu.tr
}

\author{
Hakan A. Çırpan \\ Department of ECE, \\ Istanbul Technical University, \\ Maslak, Istanbul, Turkey \\ cirpanh@itu.edu.tr
}

\begin{abstract}
For a single carrier frequency division multiple access (SC-FDMA) system, we obtain the jointly optimal power and chunk allocation policies which maximize the sum rate. Our solution is applicable to both localized and interleaved subcarrier mapping schemes. We solve the joint optimization problem by sequentially solving two sub-problems: power allocation and chunk allocation. Primarily, we use an optimal power allocation algorithm, which we derive from Karush-Kuhn-Tucker (KKT) conditions; and then we convert the optimum chunk assignment problem into a maximum weighted matching problem on a bipartite graph, and hence solve it in polynomial time. We also propose two greedy chunk allocation algorithms with lower complexity, and demonstrate that these algorithms produce near optimal results, especially for interleaved subcarrier mapping, when used in conjunction with optimal power control.
\end{abstract}

\section{INTRODUCTION}

Single carrier frequency division multiple access (SCFDMA) is becoming an increasingly popular choice for uplink transmissions, thanks to its ability to resolve the high peak to average power ratio (PAPR) issue commonly faced in orthogonal frequency division multiple access (OFDMA) systems [1], [2]. As a result, it has now entered the standards, such as LTE-Advanced, and efficient resource allocation for SCFDMA therefore remains a hot topic.

An SC-FDMA system can be considered as a pre-coded OFDMA system, whose data signals are pre-coded by a Discrete Fourier Transform (DFT) block before subcarrier mapping at the transmitter, and decoded by an Inverse DFT block after subcarrier de-mapping at the receiver [1]. In SCFDMA, the subcarriers have to be grouped into sets before being assigned to users. A set of particular subcarriers grouped together is called a chunk. While pre-coding and use of chunks reduces the PAPR compared to the OFDMA system, the intersymbol interference (ISI) rejection capability is reduced and the SC-FDMA system needs to use frequency domain equalizers to mitigate ISI [3].

The resource allocation problem for OFDMA systems has been extensively studied in the literature. A multiuser orthogonal frequency-division multiplexing (OFDM) subcarrier, bit and power allocation algorithm to minimize the total transmit power was proposed in [4]. An optimal joint subcarrier and power allocation algorithm for OFDMA systems was proposed in [5]. The joint resource allocation problem for SC-FDMA, however, has a considerably different nature than that for an OFDMA system, due the the inherent requirement that the subcarriers have to be grouped into chunks. As a result, the works on resource allocation for SC-FDMA have almost invariably focused on chunk allocation only. The problem of chunk allocation for the uplink of an SC-FDMA system with a minimum mean square error (MMSE) equalizer was considered in [3]. Yet, power allocation was not employed in [3], which assigned equal powers to all subcarriers in the same chunk and proposed greedy algorithms for chunk allocation. An optimal solution, as well as a greedy algorithm for resource allocation in uplink SC-FDMA systems were proposed in [6], without considering power allocation. In [7], the greedy solution provided in [6] was improved and three algorithms based on greedy approaches were developed: weighted sum-rate maximization, transmission with minimal number of subchannels and sum-power minimization. However, joint optimization of chunks and powers was not carried out. The solution of [3] was improved by swapping pairs of users assigned by a greedy algorithm in [8]. In [9], another modification of the greedy algorithm of [3], called maximum greedy algorithm was proposed. In [10], a virtual multiple input multiple output (V-MIMO) model was used and assuming that two users transmit their data in the same time slot and frequency band, a combination of the Hungarian algorithm and the binary switching algorithm for chunk allocation was proposed.

Unlike the previous works on resource allocation for SCFDMA that focus only on chunk allocation without power allocation, or vice-versa, in this work, we focus on joint allocation of chunks and transmit powers for uplink SCFDMA systems, and propose a jointly optimal power and chunk allocation algorithm. First, we separate the problem into two sub-problems: optimal power allocation and optimal chunk allocation. Primarily, the power allocation algorithm, which is derived from the KKT conditions, assigns the power of each of the users, to the subcarriers of each given chunk, thereby determining the rate achievable by each user on each chunk. Then a maximum weighted matching algorithm finds the matching between the users and chunks which maximizes the total rate of the system. Finally, two greedy algorithms for joint chunk and power allocation to maximize the overall sum rate are proposed.

\section{SYSTEM MODEL}

We consider an SC-FDMA system with $B \mathrm{~Hz}$ total bandwidth and $K$ users. The total frequency band is divided into $L$ subcarriers of equal bandwidth, $B / L$. The $L$ subcarriers have to be grouped into several chunks to be allocated to different users. Assuming that the system has $N$ chunks, each of the chunks have $M=L / N$ subcarriers and occupy a bandwidth of $B / N \mathrm{~Hz}$. We assume that the system is overloaded, i.e., the number of users with data available for transmission is always greater than or equal to the number of chunks. Moreover, in order to maximize the user capacity and preserve fairness, we 
assume that each user can only take one chunk in the system.

There are two types of subcarrier mapping methods in SCFDMA: localized FDMA (LFDMA) and interleaved FDMA (IFDMA). In LFDMA, the subcarriers of a chunk are adjacent to each other. In IFDMA, the subcarriers of a chunk are distrubuted equidistantly over the entire frequency band in order to avoid allocating adjacent subcarriers in deep fading. In this paper, we address both subcarrier mapping approaches.

The chunk assignment decisions are made at the base station. Thus, we assume that the base station has perfect channel state information (CSI) about the links from the users. Let $I_{n}$ denote the set of subcarriers, assigned to each chunk $n \in\{1, \ldots, N\} ; h_{i, k}$ denote the channel coefficient of user $k$ on subcarrier $i$ and $p_{i, k}$ denote the power assigned by user $k$ to subcarrier $i$. Let $\sigma_{i, k}^{2}$ be the noise power on subcarrier $i$ for user $k$. Assuming MMSE equalization is performed at the receiver, the rate achievable by each user depends on the equivalent signal-to-noise ratio (SNR) $\gamma_{n, k}$ of user $k$ on chunk $n$, obtained after MMSE equalization as in [3], [11], [12],

$$
\gamma_{n, k}=\left[\left(\frac{1}{\left|I_{n}\right|} \sum_{i \in I_{n}} \frac{\frac{p_{i, k}\left|h_{i, k}\right|^{2}}{\sigma_{i, k}^{2}}}{\frac{p_{i, k}\left|h_{i, k}\right|^{2}}{\sigma_{i, k}^{2}}+1}\right)^{-1}-1\right]^{-1} .
$$

Our goal is to obtain the optimal power and chunk allocation which jointly maximize the sum rate of the system. Note that, the rate

$$
R_{n, k}=(B / N) \log _{2}\left(1+\gamma_{n, k}\right),
$$

is achievable by user $k$, assuming that it transmits on chunk $n$. Due to the orthogonality of the chunks, the sum rate of the system can simply be computed by adding the rates achievable on each chunk, i.e.,

$$
\begin{aligned}
R_{\text {sum }} & =\sum_{n=1}^{N} \sum_{k=1}^{K} \omega_{n, k} R_{n, k}, \\
& =\sum_{n=1}^{N} \sum_{k=1}^{K} \omega_{n, k}(B / N) \log _{2}\left(1+\gamma_{n, k}\right),
\end{aligned}
$$

where $\omega_{n, k} \in\{0,1\}$ is an indicator variable, which takes the value 1 if $n$th chunk is allocated to user $k$, and 0 otherwise. Since each user is assumed to be assigned at most one chunk, we need $\sum_{n} \omega_{n, k} \leq 1$, for all $k \in\{1, \cdots, K\}$.

Subcarriers $I_{n}$ of chunk $n$ can be either consecutive or equidistantly distributed over the entire bandwidth, hence the problem we solve in this paper is applicable to both localized and interleaved chunk allocations. In the following section, we give the problem formulation and the optimality conditions to maximize sum rate of the system.

\section{Joint Power And Chunk Allocation}

Plugging (1) into (4), and after some manipulation, we can rewrite the sum rate in terms of the powers $p_{i, k}$ :

$$
R_{\text {sum }}=-\left(\frac{B}{N}\right) \sum_{n=1}^{N} \sum_{k=1}^{K}\left[\omega_{n, k}\right.
$$

$$
\left.\log _{2}\left(1-\frac{1}{\left|I_{n}\right|} \sum_{i \in I_{n}} \frac{p_{i, k}\left|h_{i, k}\right|^{2}}{p_{i, k}\left|h_{i, k}\right|^{2}+\sigma_{i, k}^{2}}\right)\right] .
$$

For simplicity, let us define

$$
c_{i, k}=\frac{\sigma_{i, k}^{2}}{\left|h_{i, k}\right|^{2}},
$$

which can be interpreted as the inverse of the normalized channel gain. Then, dropping the constant $(B / N)$, the problem of maximizing (5) is equivalent to

$$
\begin{aligned}
\max _{\substack{\omega_{n, k} \\
p_{i, k}}} \sum_{n=1}^{N} \sum_{k=1}^{K}\left[-\omega_{n, k} \log _{2}\left(1-\frac{1}{\left|I_{n}\right|} \sum_{i \in I_{n}} \frac{p_{i, k}}{p_{i, k}+c_{i, k}}\right)\right], \\
\text { s.t. } \sum_{k=1}^{K} \omega_{n, k} \leq 1 \quad \forall n, \quad \sum_{n=1}^{N} \omega_{n, k} \leq 1, \quad \forall k \\
\quad \sum_{i=1}^{M} p_{i, k} \leq \bar{P}_{k} \quad \forall k, \quad p_{i, k} \geq 0, \quad \forall i, k,
\end{aligned}
$$

where $\bar{P}_{k}$ is the available average power of user $k$.

Note that, it is rather difficult to jointly optimize the chunks allocated to each user, and powers allocated to each chunk, since the chunk allocation problem itself is a combinatoric problem even without power allocation, and the powers clearly depend on which chunk is selected, through the channel coefficients. Therefore, in what follows, we propose a two step solution, without compromising optimality:

Proposition 1: The solution to problem (7) can be obtained by solving the two step problem

$$
\begin{aligned}
\max _{\omega_{n, k}} & \sum_{n=1}^{N} \omega_{n, k} \sum_{k=1}^{K} \max _{p_{i, k}}\left[-\log _{2}\left(1-\frac{1}{\left|I_{n}\right|} \sum_{i \in I_{n}} \frac{p_{i, k}}{p_{i, k}+c_{i, k}}\right)\right], \\
\text { s.t. } & \sum_{k=1}^{K} \omega_{n, k} \leq 1 \quad \forall n, \quad \sum_{n=1}^{N} \omega_{n, k} \leq 1, \quad \forall k \\
& \sum_{i=1}^{M} p_{i, k} \leq \bar{P}_{k} \quad \forall k, \quad p_{i, k} \geq 0, \quad \forall i, k
\end{aligned}
$$

Proof: First, we fix the chunk allocation coefficients, i.e., $\omega_{n, k}$, to an admissible set that satisfy the conditions in (7). The key here is to observe that, fixing $\omega_{n, k}, \forall n$ is equivalent to fixing the set of subchannels, say $I_{n, k}$, to be allocated to each user $k$. But then, we can find the overall sum rate achievable by the users under each fixed chunk assignment by solving

$$
\begin{gathered}
\max _{p_{i, k}} \sum_{k=1}^{K}\left[-\log _{2}\left(1-\frac{1}{\left|I_{n, k}\right|} \sum_{i \in I_{n, k}} \frac{p_{i, k}}{p_{i, k}+c_{i, k}}\right)\right], \\
\text { s.t. } \quad \sum_{i \in I_{n, k}} p_{i, k} \leq \bar{P}_{k} \quad \forall k, \quad p_{i, k} \geq 0, \forall i, k .
\end{gathered}
$$

Due to the orthogonality of the subchannels, the maximization can be carried out separately over each chunk, or equivalently, over each user. Hence, the maximum operation can be moved 
inside the summation over the users, to yield

$$
\begin{gathered}
\max _{p_{i, k}}\left[-\log _{2}\left(1-\frac{1}{\left|I_{n, k}\right|} \sum_{i \in I_{n, k}} \frac{p_{i, k}}{p_{i, k}+c_{i, k}}\right)\right], \\
\text { s.t. } \quad \sum_{i \in I_{n, k}} p_{i, k} \leq \bar{P}_{k} \quad \forall k, \quad p_{i, k} \geq 0, \forall i, k .
\end{gathered}
$$

Let us denote by $R_{n, k}^{*}$, the maximum achievable rate by each user $k$ over chunk $n$, obtained from (10). Then, (7) becomes

$$
\begin{aligned}
& \max _{\omega_{n, k}} \sum_{n=1}^{N} \omega_{n, k} \sum_{k=1}^{K} R_{n, k}^{*} \\
& \text { s.t. } \sum_{k=1}^{K} \omega_{n, k} \leq 1 \quad \forall n, \quad \sum_{n=1}^{N} \omega_{n, k} \leq 1, \quad \forall k
\end{aligned}
$$

which is equivalent to (8), since the optimal chunk allocation is in the feasible set, and an exhaustive search over all chunk allocations will clearly yield the global optimum sum rate.

In what follows, we will first solve the inner maximization problem (10), derive the optimum power allocation policy for a fixed chunk assignment, and propose an algorithm to find the optimal power distribution. Then, we will solve the outer maximization problem in (8), and propose efficient optimal and suboptimal algorithms for chunk allocation.

\section{A. Optimal Power Allocation}

We start by noting that the cost function in (10) is concave, and the constraints form a convex set. Hence, this is a well defined convex optimization problem, with the solution given in the following proposition.

Proposition 2: The optimal power allocation for user $k$ over each subcarrier $i$ is given by

$$
p_{i, k}=\left(\sqrt{\frac{c_{i, k}}{\lambda_{k}}}-c_{i, k}\right)^{+},
$$

where $\lambda_{k}>0$ is a real number, selected so that the per user power constraint is satisfied, and $(\cdot)^{+}$denotes $\max (\cdot, 0)$.

Proof: Due to the convex nature of the problem, KKT conditions are necessary and sufficient for optimality. Let us define the Lagrangian by

$$
\begin{aligned}
\mathcal{L}=- & \log _{2}\left(1-\frac{1}{\left|I_{n, k}\right|} \sum_{i \in I_{n, k}} \frac{p_{i, k}}{p_{i, k}+c_{i, k}}\right) \\
& +\sum_{k=1}^{K} \xi_{i, k} p_{i, k}-\mu_{k} \sum_{k=1}^{K}\left(p_{i, k}-\bar{P}_{k}\right),
\end{aligned}
$$

where $\mu_{k}$ is the Lagrange multiplier assigned to the power constraint and $\xi_{i, k}$ are the Lagrange multipliers assigned to the non-negativity constraints for the powers. Taking partial derivatives, the KKT conditions,

$$
\begin{aligned}
\left(\frac{1}{A}\right) \frac{c_{i, k}}{\left(p_{i, k}+c_{i, k}\right)^{2}}-\mu_{k}+\xi_{i, k} & =0, \quad \forall i, k, \\
p_{i, k} \xi_{i, k} & =0, \quad \forall i, k,
\end{aligned}
$$

$$
\sum_{i \in I_{n, k}} p_{i, k} \leq \bar{P}_{k}
$$

are obtained, where,

$$
A=\ln 2\left(1-\frac{1}{\left|I_{n, k}\right|} \sum_{i \in I_{n, k}} \frac{p_{i, k}}{p_{i, k}+c_{i, k}}\right) .
$$

Letting $\mu_{k} A=\lambda_{k}$, and combining (14) and (15), the optimal powers should satisfy

$$
\frac{c_{i, k}}{\left(p_{i, k}+c_{i, k}\right)^{2}} \leq \lambda_{k}
$$

with equality if and only if $p_{i, k}>0$ (or else, $\xi_{i, k}>0$ and we get strict inequality). Solving (18) for $p_{i, k}$, and selecting $\lambda_{k}$ so that (16) are satisfied, we obtain the desired result.

The optimal power allocation can be approximately found to within any desired precision using a binary search over the real number $\lambda_{k}$, by selecting an appropriate stopping criterion. In what follows, we show that the exact optimal solution may also be found with very low complexity. First, we prove a useful property of the optimal powers:

Proposition 3: Let $c_{[i], k}$ denote an ordered version of the inverse normalized channel gains $c_{i, k}$, i.e., let $c_{[i], k}<c_{[i+1], k}$, $\forall i, \forall k$. Then, $p_{[i+1], k}>0$ implies $p_{[i], k}>0$. Moreover, if $p_{[i+1], k}>0$, then,

$$
p_{[i], k}=\sqrt{\frac{c_{[i], k}}{c_{[i+1], k}}}\left(p_{[i+1], k}+c_{[i+1], k}\right)-c_{[i], k},
$$

Proof: The first part is easily proved by contradiction. Assume $p_{[i+1], k}>0$ is possible when $p_{[i], k}=0$. Then, from (18) we need

$$
\frac{1}{\left(c_{[i], k}\right)} \leq \lambda_{k}=\frac{c_{[i+1], k}}{\left(p_{[i+1], k}+c_{[i+1], k}\right)^{2}}<\frac{1}{\left(c_{[i+1], k}\right)},
$$

which is a contradiction, as by assumption $c_{[i], k}<c_{[i+1], k}$, thereby proving the first statement. The second statement follows, since $p_{[i+1], k}>0$ implies $p_{[i], k}>0$ by the previous statement, and from (18), we have

$$
\lambda_{k}=\frac{c_{[i], k}}{\left(p_{[i], k}+c_{[i], k}\right)^{2}}=\frac{c_{[i+1], k}}{\left(p_{[i+1], k}+c_{[i+1], k}\right)^{2}} .
$$

Solving for $p_{[i], k}$, and noting that the solution is always positive, we get the desired result.

Remark 1: In plain terms, Proposition 3 states that a subcarrier in a chunk can be assigned a non-zero power, only if all subcarriers with stronger channel conditions in the chunk are already used, which is quite intuitive. Note however that, this does not mean the assigned powers have to be monotone increasing in channel gains as in the typical waterfilling solution for OFDMA systems: this interesting observation can be verified by simply considering a 2 -subcarrier per chunk scenario and setting $c_{1, k}=1, c_{2, k}=2, \lambda_{k}=0.01$ and $\bar{P}_{k}=10 \sqrt{2}+7$, which can be shown to satisfy the KKT conditions with powers $p_{1, k}=9<10 \sqrt{2}-2=p_{2, k}$.

Proposition 3 suggests a natural method for solving the optimization problem exactly without having to search for the real 
valued $\lambda_{k}$. Let $p_{[i], k}>0$ for $i=\{1, \ldots, m\}$, and $p_{[i], k}=0$ for $i=\{m+1, \ldots, M\}$. It is easy to show that by iterated use of (19), all powers can be written in terms of the first non-zero power in the sequence, i.e., $p_{[m], k}$, by

$$
p_{[i], k}=\sqrt{\frac{c_{[i], k}}{c_{[m], k}}}\left(p_{[m], k}+c_{[m], k}\right)-c_{[i], k}, \quad i=\{1, \ldots, m\}
$$

and substituting this in the power constraint, we get

$$
p_{[m], k}=\frac{\bar{P}_{k}+\sum_{i=1}^{m}\left(c_{[i], k}-\sqrt{c_{[i], k} c_{[m], k}}\right)}{\sum_{i=1}^{m} \sqrt{c_{[i], k} / c_{[m], k}}} .
$$

Once $p_{[m], k}$ is computed, all other powers can be computed recursively. Note that, one still needs to find the value of $m$ for which $p_{[i], k}>0$ for $i=\{1, \ldots, m\}$ and $p_{[i], k}=0$ for $i=\{m+1, \ldots, M\}$, but since the search space is integers, this can simply be done in at worst $M$ steps (complexity of $\log M$ is also possible by binary search, but we focus on a linear search to keep the algorithm concise), by computing $p_{[m], k}$ using (23), until a positive value is found. The overall algorithm that is used to find optimal powers is summarized as Algorithm 1.

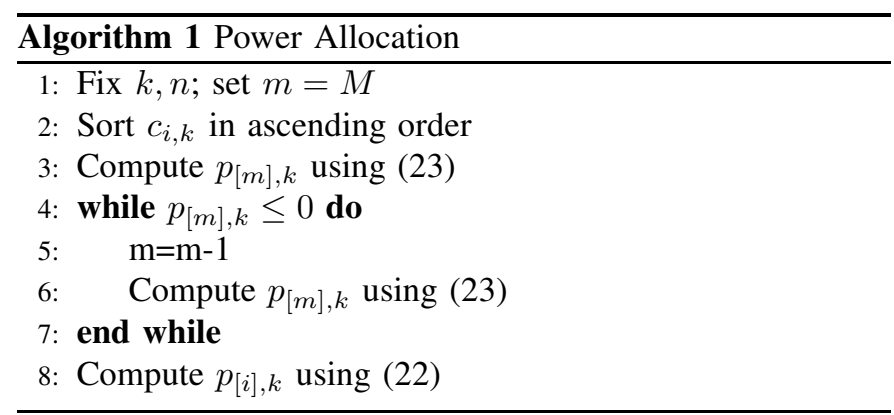

The worst case complexity of the algorithm is $O(M \log M)$, due to the channel sorting operation. Since $M=12$ in a typical SC-FDMA system, the convergence is very fast.

\section{B. Optimal and Suboptimal Chunk Allocation}

We now turn to the problem (11), and focus on optimal chunk allocation. Assume that, using Algorithm 1, the optimal power allocation, and hence the maximum achievable rate $R_{n, k}^{*}$ is computed for all possible user-chunk pairs $n, k$. The key is to realize that, since each chunk can be assigned to only one user, and vice versa, (11) can be stated as a maximum weighted matching problem on a bipartite graph, or in other words an assignment problem, where the weight on the edge connecting each user and chunk is the corresponding power optimized rate, as shown in Figure 1. Then, standard techniques from graph theory, such as the Hungarian algorithm, can be used to solve (11) in polynomial time, i.e., $O\left(\max (N, K)^{3}\right)$ and obtain $\omega_{n, k}$ that are jointly optimal with the powers found in Section III-A.

In order to further speed up the chunk assignment problem, we also propose two suboptimal greedy algorithms. The first one, which we call jointly greedy user-chunk allocation, first obtains $R_{n, k}^{*} \forall k, n$, and then finds the pair $\{\tilde{k}, \tilde{n}\}$ with the

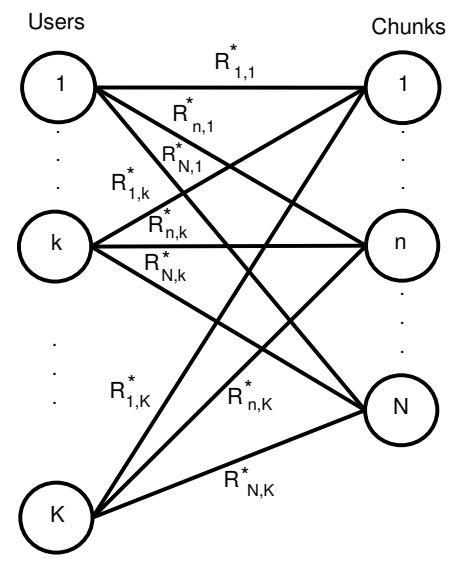

Fig. 1. Bipartite graph representing matching of users and chunks

highest rate. Next, $\tilde{k}$ and $\tilde{n}$ are deleted from the set of available chunks and users, and the search is repeated until all chunks are allocated. The complexity of this algorithm, which is given as Algorithm 2, is $O\left(N^{2} K\right)$, which is less than that of maximum weighted matching, especially with $N<K$.

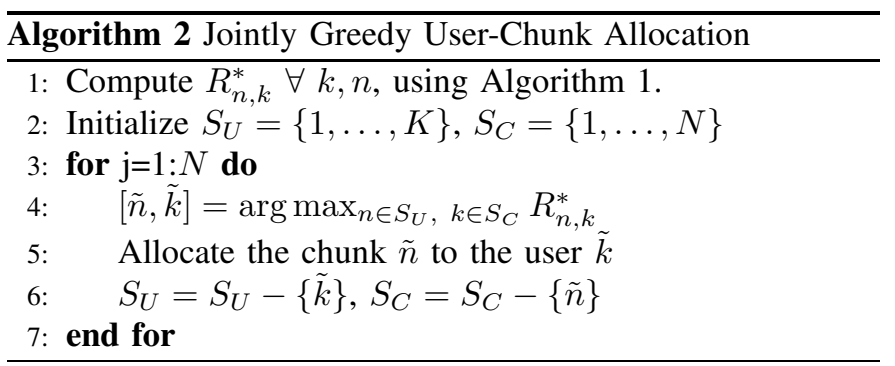

The second greedy algorithm that we propose, which is called greedy user allocation, is a much faster algorithm which simply goes through the chunks only once, and for each chunk, finds the most favorable user among the set of unassigned users, and assigns it to the chunk being considered. The pseudocode of the algorithm is given as Algorithm 3.

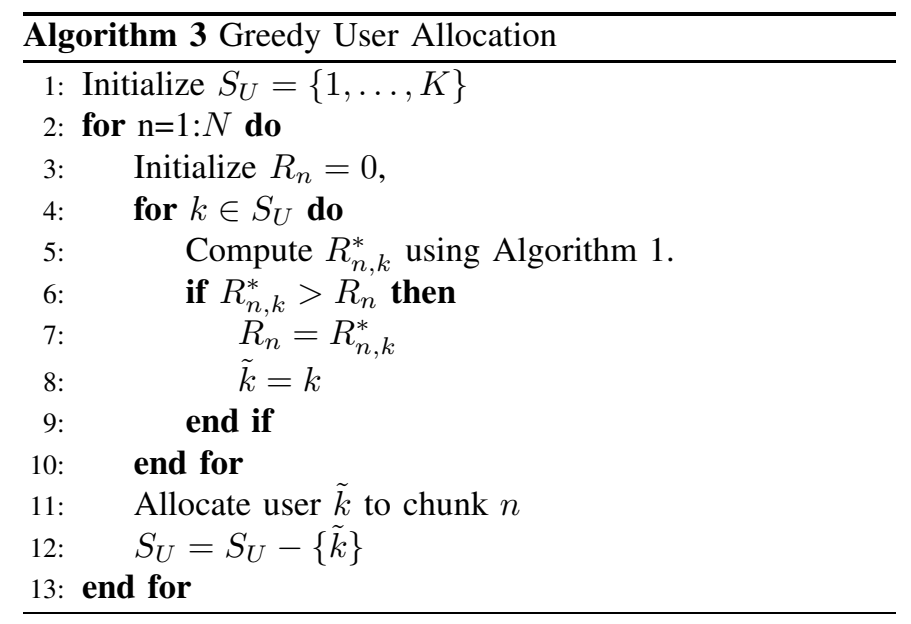

The advantage of this algorithm is twofold: it not only runs with much less complexity, i.e., $O(N K)$, but it also can be implemented such that the power optimized rates $R_{n, k}^{*}$ are computed and ordered on the fly while assigning users 
to chunks (note that only the maximum for each chunk is needed). Hence, the storage requirement is significantly less, as all user-chunk pairs need not be considered. In the following section, we compare the performance of our proposed optimal and suboptimal joint power allocation algorithms.

\section{Simulation Results}

We generate 8-tap Rayleigh channels for each user. Each tap has $10 \mathrm{~ms}$ delay, so the maximum delay spread is $70 \mathrm{~ms}$ for each user. An additive white Gaussian noise (AWGN) with zero mean is assumed at the receiver. An MMSE equalizer is used and the maximum transmit power of each user is scaled to give a average received SNR value of $-5 \mathrm{~dB}$. We assume that each chunk has $M=16$ subcarriers and each subcarrier has $19.531 \mathrm{kHz}(5 \mathrm{MHz}$ bandwidth/256 subcarriers) tone spacing.

In Figure 2, we evaluate the performance of our proposed optimal and suboptimal algorithms for joint chunk and power allocation using Monte Carlo approach, for a system with 16 chunks. We compare our results to [3], which uses equal power for each subcarrier; and two round robin scheduling schemes, labeled R-LFDMA and R-IFDMA. In curves labeled LFDMA, chunks with adjacent subcarriers are assigned to users, and in curves labeled IFDMA, equidistantly distributed subcarriers along the entire bandwidth are assigned. Although throughout the paper we assumed $K>N$, the case with $K \leq N$ was also simulated for completeness, with some necessary modifications to the algorithms. As the number of users is increased, all chunk allocation schemes except for the random round robin scheduling achieve increasing rates, which is due to the diversity created by the additional users. It is evident that localized subcarrier mapping has higher sum rate than interleaved subcarrier mapping in all cases. This is expected as interleaved subcarrier allocation creates roughly equivalent conditions for all users in each chunk, and the gain of chunk allocation will be less than that on localized FDMA where some users are more likely to experience stronger channels on some chunks due to the fading model with memory. The optimal maximum weighted matching algorithm, and the slightly less complex jointly greedy power user assignment achieve almost identical results, and the greedy user allocation performs nearly as well at much lower complexity, especially for the IFDMA scenario. The gain from optimum power allocation is much more pronounced for IFDMA with independent subcarrier fading, as in LFDMA, due to the correlation among the adjacent subcarriers, constant power allocation is already nearly optimal. As a result, we can conclude that power allocation is more vital for IFDMA, and chunk allocation is more vital for LFDMA. Nevertheless, their joint use always produce the best results. The simulations also show similar results for a 32 chunk scenario, which are not included here due to space constraints.

\section{CONCLUSION}

In this paper, we solved the joint chunk and power allocation problem for a SC-FDMA system with frequency domain equalization. The solution was performed in two steps, separating the power allocation and the chunk allocation steps; the latter of which was carried out using one optimal, and two suboptimal yet computationally more efficient approaches. We demonstrated that, for both localized and interleaved subcarrier mapping, employing power control in conjunction with chunk allocation results in significant rate gains over known results, especially for IFDMA. We further observed that, even with greedy algorithms for chunk allocation, near optimal solutions can be obtained, at much lower computational complexity.

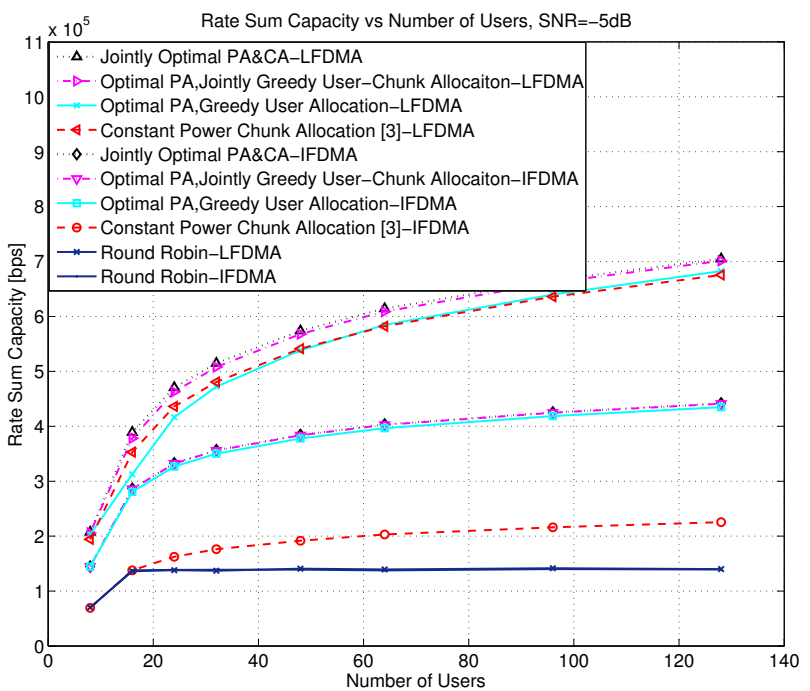

Fig. 2. Sum rate of proposed algorithms ( $\mathrm{SNR}=-5 \mathrm{~dB}, N=16$ chunks, $B=5 \mathrm{MHz}, L=256$ subcarriers).

\section{REFERENCES}

[1] H. G. Myung, J. Lim and D. J. Goodman, "Single Carrier FDMA for Uplink Wireless Transmission,” IEEE Veh. Technol., 1(3): 30-38, Sept. 2006.

[2] H. G. Myung, J. Lim and D. J. Goodman, "Peak-to-average Power Ratio of Single Carrier FDMA Signals with Pulse Shaping," IEEE PIMRC, pp.1-5, Helsinki, Finland, Sept. 2006.

[3] J. Lim, H. G. Myung, K. Oh and D. J. Goodman, "Channel-Dependent Scheduling of Uplink Single Carrier FDMA Systems," IEEE 64th Veh. Technol. Conf., VTC-2006 Fall, pp.1-5, Montreal, Canada, Sept. 2006.

[4] C. Y. Wong et al., "Multiuser OFDM with adaptive subcarrier, bit and power allocation," IEEE J. Sel. Areas Commun., 17(10): 1747-1758, Oct. 1999.

[5] K. Kim, Y. Han and S. Kim, "Joint Subcarrier and Power Allocation in Uplink OFDMA Systems," IEEE Commun. Letters, 9(6): 526-528, June 2005

[6] I. C. Wong, O. Oteri and W. McCoy, "Optimal Resource Allocation in Uplink SC-FDMA Systems," IEEE Trans. on Wireless Commun., 8(5): 2161-2165, May 2009.

[7] F. I. Sokmen and T. Girici, "Uplink Resource Allocation Algorithms for Single-Carrier FDMA Systems," European Wireless Conf $(E W)$., pp.339-345, Lucca, Italy, Apr. 2010.

[8] W. C. Pao and Y. F. Chen, "Chunk Allocation Schemes for SC-FDMA Systems," Veh. Technol. Conf., pp.1-5, Taipei, Taiwan, May 2010.

[9] O. Nwamadi, X. Zhu and A. Nandi, "Dynamic Subcarrier Allocation for Single Carrier-FDMA Systems," European Signal Processing Conf., Aalborg, Denmark, Aug. 2008.

[10] M. A. Ruder, D. Ding, U. L. Dang and W. H. Gerstacker, "Combined User Pairing and Spectrum Allocation for Multiuser SC-FDMA Transmission,” IEEE ICC, pp.1-6, Kyoto, Japan, June 2011.

[11] T. Shi, S. Zhou and Y. Yao, "Capacity of Single Carrier Systems with Frequency-Domain Equalization," IEEE 6th CAS Symp. on Emerging Technologies: Mobile and Wireless Commun., Shanghai, China, 2: 429 - 432, May 31-June 2, 2004.

[12] 3GPP R1-050718, "Simulation methodology for EUTRA UL:IFDMA and DFT-Spread-OFDMA,” Sept. 2005. 